\title{
Chromatin-Mediated Restriction of Nuclear Factor 1/CTF Binding in a Repressed and Hormone-Activated Promoter In Vivo
}

\author{
Sergey Belikov, Carolina Åstrand, Per-Henrik Holmqvist, and Örjan Wrange* \\ Department of Cell and Molecular Biology, The Medical Nobel Institute, \\ Karolinska Institutet, SE-17177 Stockholm, Sweden
}

Received 16 December 2003/Accepted 7 January 2004

\begin{abstract}
Mouse mammary tumor virus (MMTV) promoter-driven transcription is induced by glucocorticoid hormone via binding of the glucocorticoid receptor (GR). The MMTV promoter also harbors a binding site for nuclear factor 1 (NF1). NF1 and GR were expressed in Xenopus oocytes; this revealed GR-NF1 cooperativity both in terms of DNA binding and chromatin remodeling but not transcription. A fraction of NF1 sites were occupied in a hormone-dependent fashion, but a significant and NF1 concentration-dependent fraction were constitutively bound. Activation of the MMTV promoter resulted in an $\sim \mathbf{5 0}$-fold increase in the NF1 accessibility for its DNA site. The hormone-dependent component of NF1 binding was dissociated by addition of a GR antagonist; however, the antagonist RU486, which supports partial GR-DNA binding, also maintained partial NF1 binding. Hence GR-NF1 cooperativity is independent of agonist-driven chromatin remodeling. NF1 induced the formation of a micrococcal-nuclease-resistant protein-DNA complex containing the DNA segment from -185 to -55 , the MMTV enhanceosome. Coexpression of NF1 and Oct1 resulted in a significant stimulation of hormone-induced MMTV transcription and also in increased basal transcription. We propose that hormone-independent NF1 binding may be involved in maintaining transcriptional competence and establishment of tissue-specific gene networks.
\end{abstract}

The mouse mammary tumor virus (MMTV) promoter is strongly induced by glucocorticoid hormone via binding of the glucocorticoid receptor (GR) (43) to a cluster of glucocorticoid-responsive elements (GREs) (35). In addition, binding sites for several other transcription factors have been identified, such as those for nuclear factor 1 (NF1) (34) and octamer binding factor 1 (Oct1) $(9,47)$. The functional importance of the NF1 binding site was demonstrated by the drastic decrease in basal as well as hormone-induced transcription in mutants involving the NF1 site $(11,13,47)$. The 1.2-kb MMTV long terminal repeat (LTR) appears to be organized into six translationally positioned nucleosomes in vivo (42). However, the reconstitution of glucocorticoid hormone-activated MMTV transcription in Xenopus oocytes showed the translationally positioned nucleosomes to be hormone dependent and hence to occur only in the activated state (5). The six positioned nucleosomes in the MMTV LTR are usually designated A to F, where B organizes the cluster of GREs. This nucleosome also coincides with a hormone-inducible DNase I hypersensitive domain (49). The same segment of hypersensitivity is developed by $\mathrm{OH}$ radical footprinting $(5,42)$. This implies that the nucleosome $\mathrm{B}$ chromatin is changed into a more accessible state during glucocorticoid hormone activation. It has been reported that the increase in DNA accessibility during gene activation allows access of other constitutive transcription factors to their cognate binding sites, one such constitutive DNA binder being NF1 $(15,48)$. This important finding led to the concept of a GR-mediated chromatin opening, thus allowing

\footnotetext{
* Corresponding author. Mailing address: Dept. of Cell and Molecular Biology, The Medical Nobel Institute, Box 285, Karolinska Institutet, SE-17177 Stockholm, Sweden. Phone: 468728 7373. Fax: 46831 35 29. E-mail: orjan.wrange@cmb.ki.se.
}

the access of NF1 to its binding site. In vitro reconstitution of nucleosomes onto the MMTV promoter GRE DNA indicated that the NF1 protein is less prone to bind its cognate DNA site in a nucleosome $(4,8,38)$. GR, on the other hand, has a remarkable capacity to invade a nucleosome and bind to its DNA site $(28,36)$. The different arrangements of the proteinDNA contact points may explain this difference. Biochemical studies imply that NF1 forms contacts with DNA on different sides along the DNA axis (18), while the crystal structure of the GR-DNA complex revealed contacts in two consecutive major grooves from one side along the DNA axis (29).

$\mathrm{NF} 1$, also referred to as NF1/CTF, is ubiquitously expressed in most tissues and possesses a constitutive DNA-binding capacity. It was first discovered as a DNA replication factor for adenovirus (33) and has been subsequently found in a large and growing number of gene regulatory elements both in viral enhancers and in cellular genes (reference 21 and references therein). NF1 binds to its DNA site as a homodimer to the symmetric consensus sequence TTGGC(N5)GCCAA. The NF1 gene family of vertebrates contains four different, albeit highly related, genes, the NF1-A, NF1-B, NF1-C, and NF1-X genes. The DNA-binding and dimerization domain, of about 200 amino acids, is strongly conserved in all four NF1 proteins, while the C-terminal half is more variable. The NF1-C protein contains a proline-rich domain that was shown to stimulate transcription (32). There are several different splice variants from each of the four NF1 genes, and they are differently expressed in various tissues (21). Protein domains that mediate transcriptional repression have been identified in both NF1-A and NF1-X. It is likely that repression and activation by NF1 proteins will be both cell type and context specific. Disruption of the NF1-C gene in mice demonstrated an essential role in tooth root development (46), disruption of the NF1-B gene 
resulted in severe lung hypoplasia (22), and disruption of the NF1-A gene resulted in drastic neuroanatomical defects and perinatal lethality (16).

The concept of how different classes of transactive DNA binding factors can bind to their target DNA sites and affect the binding of other constitutive-DNA-binding factors is of primary importance for the mechanistic understanding of the transcriptional induction event. In vitro studies have suggested that the packaging of DNA into nucleosomes increases the cooperative effects of transactive factor binding, thus resulting in improved binding of a second factor $(1,3)$ or several copies of the same factor (36). However, in vivo studies are required in order to understand how these events occur on the chromatinized template. We used GR-induced MMTV transcription in Xenopus oocytes to address these issues (5). The apparent absence of endogenous NF1 in Xenopus oocytes (see Results) opened the possibility of monitoring the effect of exogenously expressed NF1 protein. This allowed us to address the DNA accessibility of this constitutive DNA binder as a function of hormone induction and intranuclear NF1 concentration. These experiments showed that NF1 is able to bind its DNA site also in the absence of GR induction, albeit with an apparent $\sim 50$ fold-lower affinity than the hormone- and GR-activated promoter. GR cooperated with NF1, resulting in increased restriction enzyme accessibility of a SacI site located near the center of the B nucleosome. NF1 also enhanced GR-DNA binding, but NF1 did not significantly affect hormone-induced MMTV transcription. Furthermore, the GR-dependent component of NF1 binding was reversible and followed GR binding, even when mediated by a hormone antagonist, rather than reflecting hormone agonist-dependent transcription. In addition, NF1 altered the GR- and hormone-dependent enhanceosome-subnucleosome structure as developed by DNase I or micrococcal nuclease (MNase). Finally NF1 was shown to significantly stimulate transcription when coexpressed with another ubiquitous transcription factor, i.e., Oct1.

\section{MATERIALS AND METHODS}

Plasmids. pMMTV:M13 harbors the 1.2-kb MMTV LTR fused to the herpes simplex virus thymidine kinase gene at position +137 of the MMTV promoter (11). The human Oct1/RN3P vector for Oct1 mRNA production was generated from PCR-amplified human Oct1 cDNA of pGSOct-1+ (27) with $0.5 \mu \mathrm{M}$ (each) primers 5'GTCAGGATCCACAGCCACCATGAACAATCCGTCAGAAAC and 5'GTCAGCGGCCGCTCACTGTGCCTTGGA. The PCR product and RN3P vector DNA (50) were both cleaved with BamHI and NotI and purified with QIAquick (Qiagen) from a SeaPlaque GTG agarose gel (BioWhittaker Molecular Applications, Rockland, Maine), followed by ligation and plasmid preparation according to standard procedures.

The plasmid was linearized with Asp718 and in vitro transcribed by the mMESSAGE mMACHINE kit (Ambion). The pigNF1-C1/RN3P vector was constructed in the similar fashion by using the primers 5'GTCAGAATTCACA GCCACCATGATTCGTCCCCGCTCTG and 5'GTCAGCGGCCGCTCATCC CAGATACCAGGACT. The PCR template was the pigNF1-C1 cDNA in plasmid pAA-CTF1 (39). The PCR product was trimmed with EcoRI and NotI and ligated into RN3P vector DNA (50) that had been cleaved with the same two enzymes. pigNF1-C1/RN3P was linearized and in vitro transcribed as described above. Murine NF1-B2/RN3P was constructed as described above with primers 5'GTCAGGATCCACAGCCACCATGATGTATTCTCCCATCTGTCTC and 5'GTCAGCGGCCGCTCAGCCCAGGTACCAGGACTGGC and the murine NF1-B2 cDNA from pCHNF-B (14). The ligated mouseNF1-B2/RN3P construct was linearized by SfiI, and then the $3^{\prime}$ overhang was filled with Klenow fragments before in vitro transcription. The construction of $\mathrm{p} \beta \mathrm{GR} / \mathrm{RN} 3 \mathrm{P}$ for production of mRNA for rat GR was described before (5).

Oocyte injections. Oocyte injections were as described previously (5).
Quantification of MMTV transcription. Quantification of MMTV transcription by S1 nuclease and DNA analysis were as described previously (19) with the exception that a synthetic oligonucleotide identical to the lower strand of the -6 -to- +64 DNA segment of the MMTV LTR was labeled with $\left[\gamma-{ }^{33} \mathrm{P}\right]$ ATP (Amersham Biosciences) and $\mathrm{T}_{4}$ polynucleotide kinase and used as a probe. Furthermore, the oocyte homogenate was split in two aliquots, and then RNA was quantified in one half and the amount of injected pMMTV:M13 reporter DNA was quantified in the other aliquot by primer extension as for the SacI accessibility assay (see below). Then transcription was expressed as the ratio of MMTV RNA/MMTV DNA in relative terms since there is a linear correlation between injected DNA and hormone-activated MMTV transcription, at least up to $5 \mathrm{ng}$ of injected pMMTV:M13 reporter single-stranded DNA (ssDNA) (P.-H. Holmqvist and Ö. Wrange, unpublished data). The analysis was done in duplicate to monitor experimental variation. We previously used an adenovirus major late (AdML) reporter construct as a reference for MMTV RNA quantification (5). Here we found that systematic variation of AdML transcription was dependent both on hormone treatment and on the presence of injected NF1 and Oct1. Hence the AdML construct could not be used as a transcriptional reference when small differences in transcriptional activity were to be analyzed (data not shown).

Intranuclear GR and NF1 concentration. Oocytes were injected with RNA coding for GR and NF1 proteins and placed in oocyte medium OR2 (82.5 mM $\mathrm{NaCl}, 2.5 \mathrm{mM} \mathrm{KCl}, 1 \mathrm{mM} \mathrm{CaCl}_{2}, 1 \mathrm{mM} \mathrm{MgCl} 2,1 \mathrm{mM} \mathrm{Na}_{2} \mathrm{HPO}_{4}, 5 \mathrm{mM}$ HEPES [pH 7.8]), also containing $\left[{ }^{35} \mathrm{~S}\right]$ methionine at $1,000 \mathrm{Ci} / \mathrm{mmol}$ (Amersham) at a concentration of $0.02 \mu \mathrm{Ci} / \mu \mathrm{l}$ of medium overnight. Then the nuclei were dissected in pools of five and analyzed by sodium dodecyl sulfate- $12 \%$ polyacrylamide gel electrophoresis (20). Relative amounts GR and NF1 were estimated by quantification on a Fuji BAS-2500 bioimaging analyzer using Image Gauge, version 3.3, software with correction for the methionine content in the respective proteins. An aliquot was also analyzed by immunoblotting with GR antiserum together with known amounts of rat GR purified from rat liver as described previously (36) to serve as a standard curve for calculation of absolute amounts of NF1 that followed a linear correlation between injected RNA and translated proteins (C. Åstrand and Ö. Wrange, unpublished data).

DMS in vivo footprinting and primer extension. Dimethyl sulfate (DMS) in vivo footprinting and primer extension were described before (6). For the DMS in vivo footprinting, depurinization was achieved by a 15 -min incubation at $90^{\circ} \mathrm{C}$ and then DNA cleavage was triggered by a 30-min incubation in $0.09 \mathrm{M} \mathrm{NaOH}$ at $90^{\circ} \mathrm{C}$. This protocol results in preferable methylation and cleavage of guanines but also, to some extent, of adenines (30). The method was used to monitor specific binding of GR and NF1 based on previously revealed patterns of methylation protection of guanosines $(6,7)$.

MNase, DNase I, and SacI accessibility assays. The MNase, DNase I, and SacI accessibility assays were as described previously (5).

\section{RESULTS}

NF1 expressed in Xenopus oocyte binds to its cognate DNA site in the MMTV promoter. The MMTV LTR harbors at least four binding sites for the GR (Fig. 1A), named GRE I to IV. Furthermore, it harbors one binding site for the ubiquitous NF1 (Fig. 1A) and two octamer binding sites recognized by the Oct1 transcription factor located close to the TATA box. To address the effects of NF1 on MMTV-driven transcription, we injected the pMMTV:M13 reporter construct as circular ssDNA into the nuclei of Xenopus oocytes, thus resulting in chromatin assembly during second-strand synthesis $(2,5)$. Cytoplasmic injection of in vitro-transcribed synthetic mRNA coding for rat GR alone or mixed with synthetic mRNA coding for the porcine NF1-C1 resulted in efficient protein synthesis, which was monitored by measuring $\left[{ }^{35} \mathrm{~S}\right]$ methionine incorporation (Fig. 1B, GR band of about $90 \mathrm{kDa}$ and NF1 band of about $55 \mathrm{kDa}$ ). Quantification of the incorporated radioactivity revealed a linear correlation between the amounts of injected mRNA, up to at least $12 \mathrm{ng}$ per oocyte, and the amount(s) of protein(s) (data not shown).

The in vivo binding of GR to its four binding sites (GRE I to IV; Fig. 1A) and of NF1 to its single DNA binding site in the 

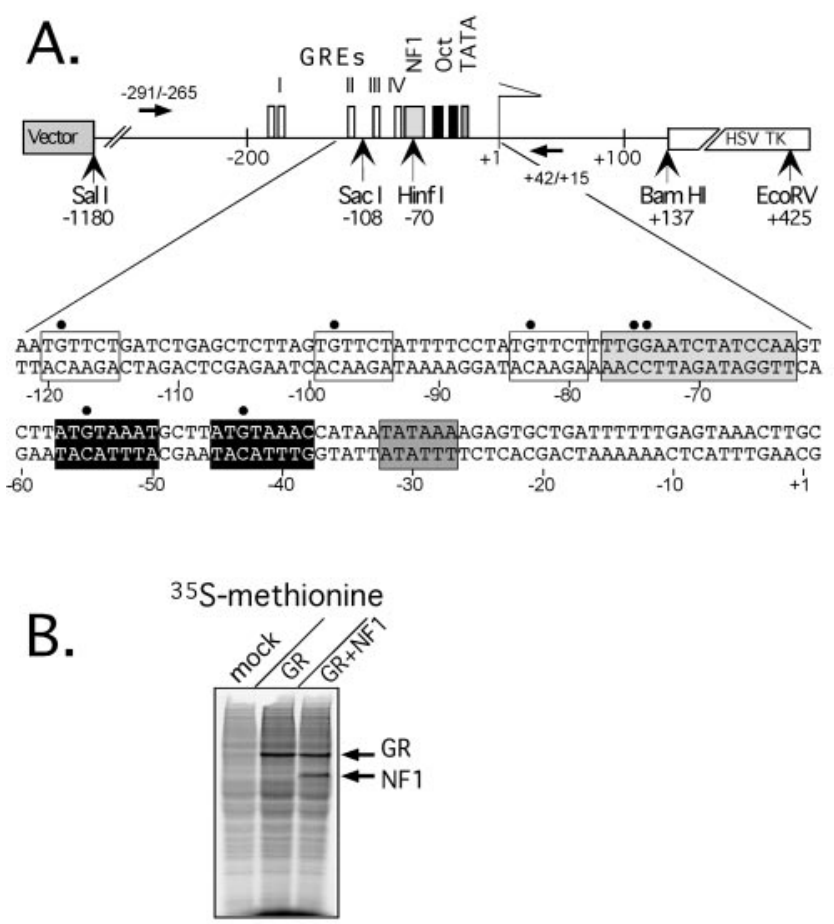

C.

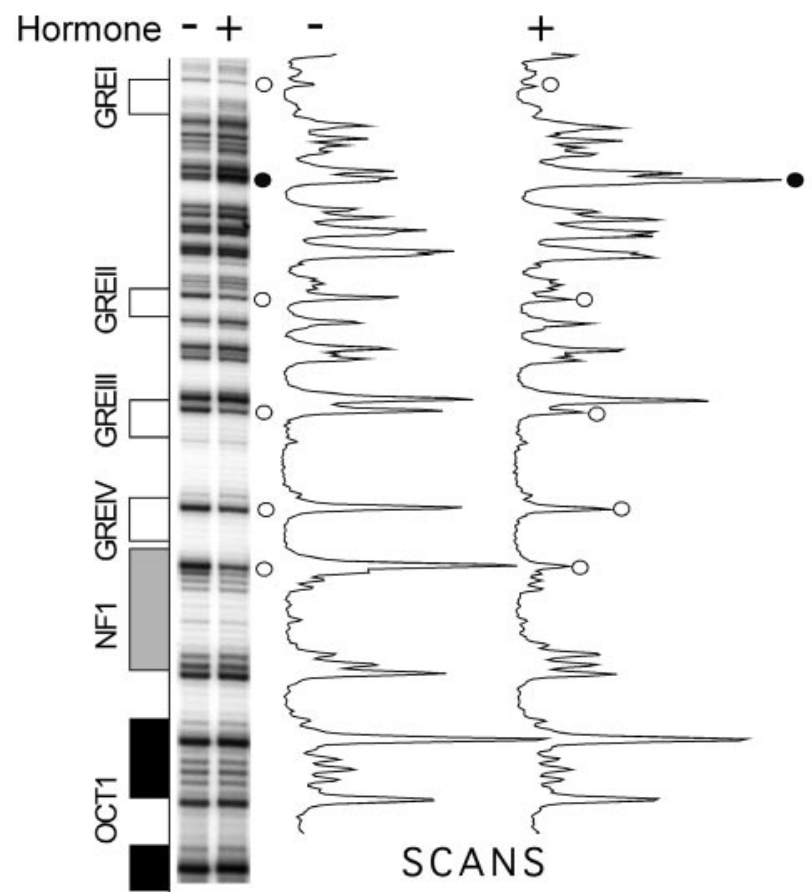

FIG. 1. (A) The reporter DNA construct pMMTV:M13 used for injection. Solid black arrows, primers used for primer extension analysis of SacI in situ accessibility and DMS methylation protection. The restriction enzyme sites shown are referred to in the text. Arrow $(+1)$, transcription start site. GRE I to IV (white boxes), the NF1 binding site (light gray), Oct1-binding sites (black), and the TATA box (dark gray) and their indicated cognate DNA binding segments are displayed with the same shading. Black dots, protected guanines in DMS in vivo footprinting. (B) Autoradiography of Xenopus oocyte extract $24 \mathrm{~h}$ after injection of $6 \mathrm{ng}$ of GR mRNA alone or together with $12 \mathrm{ng}$ of NF1 mRNA followed by incubation in $\left[{ }^{35} \mathrm{~S}\right]$ methionine (see Materials and
MMTV promoter was monitored by determining DMS methylation protection (Fig. 1C, compare lanes - and + for the hormone triamcinolone acetonide [TA] and corresponding scans). Hormone induction resulted in distinct protection of the guanines in GREs I to IV and also the doublet of guanines at -74 and -75 within the NF1 binding site, which is a hallmark for specific NF1 binding (7) (Fig. 1A). The hormoneinduced DMS methylation protection of the GREs was dependent on injection of GR mRNA; likewise, the protection at the NF1 site was dependent on NF1 mRNA injection (see further data below). There was no detectable level of endogenous GRor NF1-like DNA-binding activity in Xenopus oocytes as seen by DMS in vivo footprinting (Fig. 1; see Fig. 3). This was corroborated by the same level of methylation at corresponding guanines when naked DNA was used (data not shown).

NF1 does not significantly affect basal- or GR-dependent MMTV transcription. GR mRNA- and MMTV reporter DNAinjected oocytes show a strong hormone-dependent transcription. The MMTV transcription in the absence of hormone is low, usually below $1 \%$ of the hormone-induced transcription (5). The distinct NF1 binding to the MMTV promoter prompted us to investigate its effect on MMTV transcription. The ssDNA reporter was injected in order to allow chromatin assembly (2), and then the GR mRNA or the GR mRNA-NF1 mRNA mixture was injected. The rationale was that the NF1 protein binds constitutively to DNA (see below) and hence this injection scheme would confront the expressed NF1 protein with an already chromatinized template. The effect of NF1 on MMTV promoter-driven transcription was quantified by an S1 nuclease protection assay, where injected and recovered MMTV DNA was used as a reference for the RNA amounts in order to correct for variations in oocyte injections. The relative amounts of GR and NF1 protein were estimated by $\left[{ }^{35} \mathrm{~S}\right] \mathrm{me}-$ thionine labeling (Fig. 1B). The same amount of GR protein was produced both in the presence and absence of NF1 (not shown).

In spite of the strong binding of NF1 to the MMTV promoter (Fig. 1C), there were small and variable effects by NF1 on hormone-induced MMTV transcription. In some experiments we obtained a slight transcriptional stimulation (Fig. 2A), while in other experiments the presence of NF1 caused an inhibition (Fig. 2B). The oocytes were injected with relatively large amounts of GR and NF1 mRNA in order to achieve high enough levels to saturate their cognate binding sites on the MMTV promoter. It was conceivable that the large amounts of GR that were expressed in the oocytes might override any stimulatory effect by NF1. We have previously demonstrated that nuclear translocation, transcription, and chromatin remodeling induced by GR are completely dependent on the hormone concentration and that half-maximal transcription and receptor-DNA binding are achieved at around $10 \mathrm{nM}$

Methods). (C) DMS methylation protection analysis of the MMTV DNA segment -200 to -50 for oocytes injected with $3 \mathrm{ng}$ of pMMTV:M13 DNA and GR-NF1 mRNA as for panel B and the next day not treated $(-)$ or treated $(+)$ with $1 \mu \mathrm{M}$ synthetic glucocorticoid TA. Radioactivity scans show the two lanes with highlighted hormonedependent effects: methylation-protected bands (white circles) and a hypermethylated band (black dot). 
A.

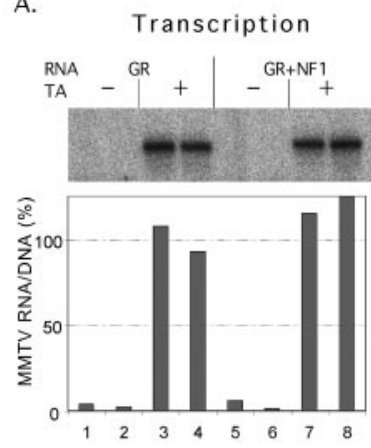

B.
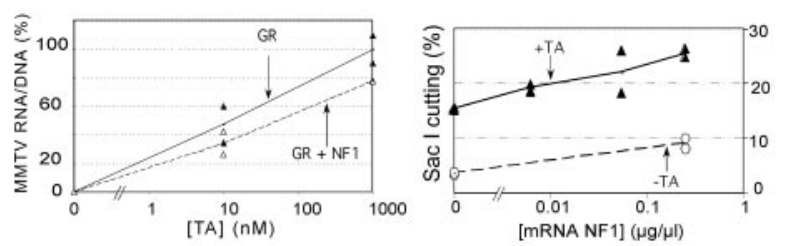

FIG. 2. Effects on MMTV transcription (A and B) and SacI in situ cutting (C and D) by GR and NF1. MMTV reporter DNA and the indicated mRNA mixtures injected into oocytes were kept for $19 \mathrm{~h}$ and then exposed to hormone (TA) for $9 \mathrm{~h}$. Shown are an S1 nuclease analysis (A) and a chromatin-remodeling assay by SacI in situ cutting (C) of double samples from the same oocyte homogenate, a pool of eight oocytes in each sample. (B) Oocytes injected as in panel A were incubated with the indicated hormone concentrations. Double samples of five oocytes each were analyzed for MMTV transcription. (D) SacI in situ cutting of double samples of seven oocytes each injected as in panel A but with the indicated amounts of mRNA for NF1 per oocyte and $3 \mathrm{ng}$ of GR mRNA. A subsaturating dose of $8 \mathrm{nM}$ hormone was added (+TA) or not added (-TA).

hormone (6) (data not shown). Hence we also evaluated the effect of NF1 at low hormone concentrations. However, several hormone titration experiments did not reveal any consistent positive or negative effect of NF1 on MMTV (Fig. 2B). The influence of different NF1 concentrations was also addressed. Oocytes were injected with low and increasing concentrations of NF1 mRNA together with a constant level of GR mRNA and then treated with both a subsaturating level and a fully saturating concentration of hormone, i.e., $10 \mathrm{nM}$ and $1 \mu \mathrm{M}$, respectively. Again there was no significant effect on MMTV transcription (data not shown). In our efforts to uncover an effect of NF1 on MMTV transcription we also reversed the injection protocol such that NF1 and/or GR mRNA was injected $5 \mathrm{~h}$ prior to the ssDNA reporter. This would allow significant levels of NF1 protein to be present during chromatin assembly. However, these conditions also failed to generate any effects by NF1 on MMTV transcription (data not shown).

NF1 is expressed from four highly related genes in vertebrates, the NF1-A, -B, -C and -X genes (see the introduction). The murine NF1-B2 was previously shown to render the most potent stimulation of MMTV transcription in a transient-transfection assay with chorion carcinoma cells (JEG-3) compared to the other three murine NF1 proteins (14). Hence we injected the murine NF1-B2 mRNA and compared its effect on glucocorticoid-mediated MMTV transcription with that of the porcine NF1-C1 in oocytes previously injected with MMTV reporter DNA. This experiment did not reveal any difference between these two NF1 variants in terms of hormone-driven MMTV transcription. Both NF1 variants were expressed to similar levels in the oocytes according to $\left[{ }^{35} \mathrm{~S}\right] \mathrm{methionine}$ labeling and sodium dodecyl sulfate-polyacrylamide gel electrophoresis analysis as described above (data not shown).

In seven independent experiments we observed a wide range of transcriptional NF1 effects, from a 1.3- to a 0.6-fold effect; hence both stimulatory and inhibitory effects were seen, but on the average there was no consistent effect by NF1 on MMTV transcription in Xenopus oocytes.

NF1 binding increases DNA accessibility as evaluated by SacI in situ cutting. We analyzed DNA accessibility by use of the restriction enzyme SacI. Its cleavage site is located at position -108 between GRE II and III (Fig. 1A). These experiments showed the usual hormone-dependent increase in SacI cutting (5), albeit it was further increased by NF1 (Fig. 2C, compare lanes 3 and 4 to lanes 7 and 8). The level of NF1mediated and hormone-dependent increase was clearly significant and increased with increasing amounts of injected NF1 mRNA (Fig. 2D). The effect of NF1 on SacI cutting in the absence of hormone was lower, but a distinct stimulation was seen at high NF1 concentrations (Fig. 2D and data not shown). There is $37 \mathrm{bp}$ of DNA between the dyads of the SacI site and the NF1 site (Fig. 1A). We conclude that NF1 binding increases DNA accessibility in the MMTV promoter at least up to this position.

Constitutive and hormone-dependent NF1 binding. NF1 has been shown to bind with high affinity to its cognate DNA site (44). We asked whether the GR- and hormone-dependent NF1 binding (Fig. 1C) could be reversed by the consecutive addition of a glucocorticoid antagonist. We previously used this protocol to reversibly induce and repress the MMTV promoter (6). The induction was mediated by the weak hormone agonist corticosterone at a limiting concentration of $0.1 \mu \mathrm{M}$. Repression was then obtained by treatment with a high concentration of a glucocorticoid antagonist. We used as antagonists either $12 \mu$ M RU486 or $3 \mu$ M RU43044, the maximal concentrations that are soluble in the oocyte medium. The oocyte injection and hormone treatment protocol is displayed in Fig. 3A. Quantification of the intranuclear MMTV promoter-driven transcript revealed a strong reduction in the antagonist-treated oocytes, thus indicating an inhibition of MMTV transcription compared to that in the oocytes where the agonist treatment was maintained (6) (data not shown).

In agreement with the results in Fig. $1 \mathrm{C}$ the in vivo footprinting by DMS methylation protection of the GR mRNAinjected oocytes showed an agonist-dependent protection for the GREs (Fig. 3B, left). This $\sim 50 \%$ methylation protection was partly reversed by the antagonist RU486 and was fully reversed by the antagonist RU43044 (Fig. 3B, compare scan A [no hormone] to scans B [agonist] and C [the two antagonists]). This confirms our previous findings that GR does indeed bind its GRE, albeit with lower affinity, in the presence of the glucocorticoid antagonist RU486 while the other glucocorticoid antagonist, RU43044, does not support any detectable GR-GRE binding (6).

GR mRNA-injected oocytes did not generate any hormonedependent DMS methylation protection at the NF1-specific -75 and -74 guanines (Fig. 3B and C, left; compare lane A to $\mathrm{B} 1$ and $\mathrm{B} 2$ in Fig. 3B), hence confirming that oocytes do not 


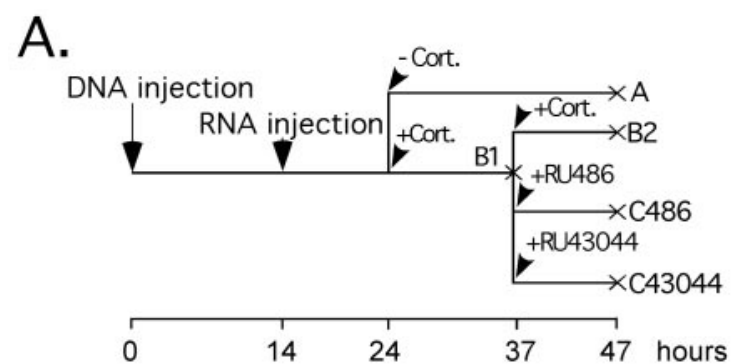

B.
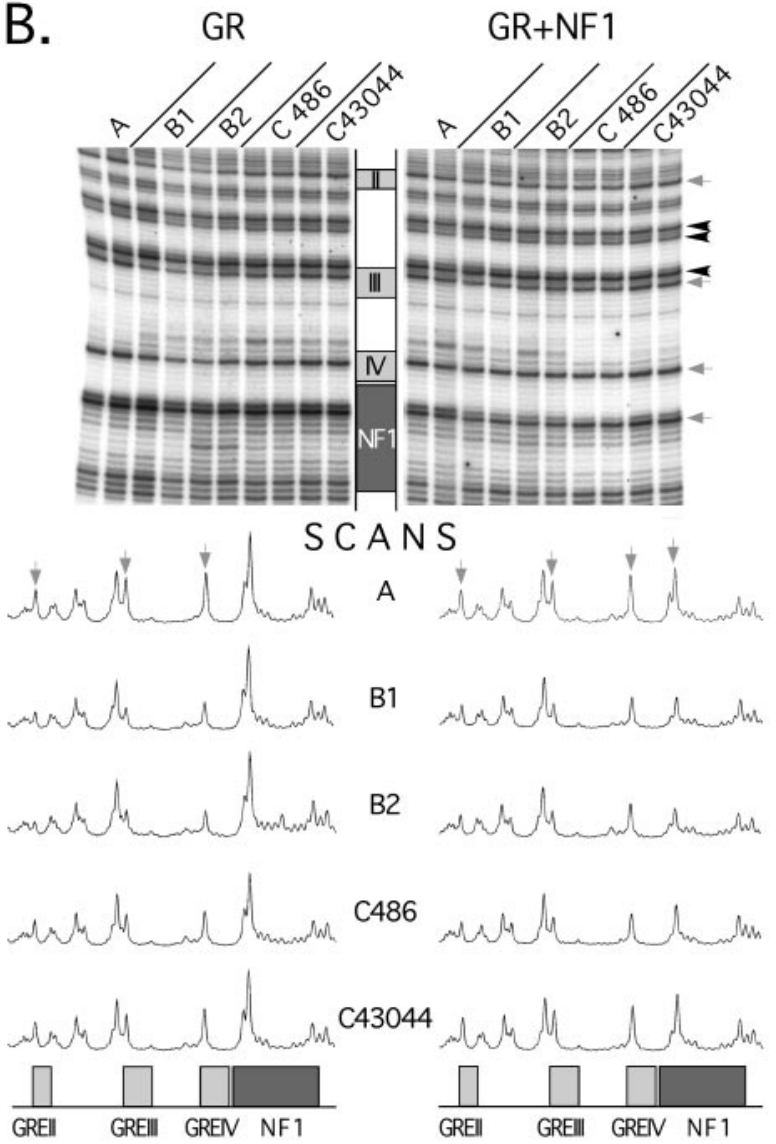

C.

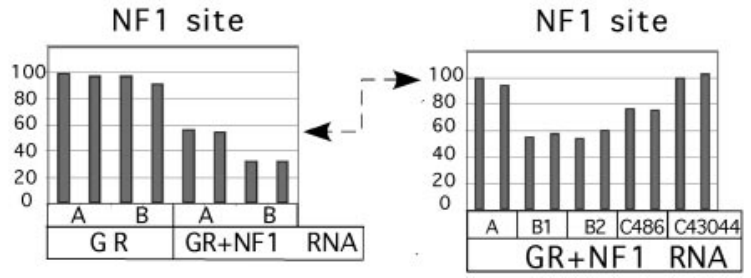

FIG. 3. GR-dependent NF1 binding is reversible. (A) Experimental design. (B) Autoradiogram of DMS in vivo footprinting developed by primer extension. Lanes: A, no hormone; B1 and B2, corticosterone agonist at time $13 \mathrm{~h}(\mathrm{~B} 1)$ and $23 \mathrm{~h}(\mathrm{~B} 2)$; C, oocytes exposed to the indicated antagonist at time $13 \mathrm{~h}$ and analyzed as double samples. Grey arrows, right, protected bands; black arrowheads, reference bands. harbor significant amounts of endogenous NF1 binding activity. The GR mRNA- and NF1 mRNA-injected oocytes on the other hand showed a distinct NF1-specific methylation protection even in the absence of hormone (Fig. 3B, right, and $\mathrm{C}$, left). In this case the mock-treated oocytes showed only $56 \%$ methylation at the NF1 site compared to the GR mRNA-injected controls. The NF1 protein is thus able to bind to its cognate DNA site also in the absence of hormoneinduced and GR-dependent chromatin remodeling. This hormone-independent NF1 binding did not generate any significant stimulation of transcription (Fig. 2A) (our unpublished observations). However, treatment with the hormone agonist resulted in a further increase in NF1 binding, as revealed by enhanced DMS methylation protection, rendering $33 \%$ methylation (Fig. 3B, right scans B1 and B2, and C, left).

When the level of DMS methylation of NF1-specific guanines in the absence of hormone was used as control, i.e., $100 \%$, then hormone induction resulted in $58 \%$ methylation at the NF1 site (Fig. 3C, right). The addition of RU486 resulted in partial reduction of NF1 protection to $77 \%$ of methylation, while addition of RU43044 rendered a 102\% methylation. Hence the agonist-dependent fraction of NF1 binding is reversible. Similar results were obtained in three independent experiments (not shown). We note that the partial binding of the RU486-GR complex to the GRE (Fig. 3B, lane C 486) seemed to correlate to a partial binding of the NF1 to its cognate DNA site (Fig. 3B, lane C 486 and C, right). We previously showed that chromatin structural changes occurring in the MMTV promoter during agonist-mediated induction are fully reversed by RU486 in parallel with the reduction in transcription (6). The occurrence of partial NF1 binding in the presence of partial GR-GRE binding mediated by RU486 implies that NF1-DNA binding is coupled to GR-DNA binding as such rather than to GR agonistinduced chromatin remodeling and transcription.

We conclude that the NF1 binding site is partly occupied by NF1 in the absence of hormone induction while another fraction of the binding sites display a hormone-dependent NF1 binding. The latter fraction are reversibly bound and dissociated on the sequential addition of a GR agonist followed by an antagonist. Astonishingly, the reversible NF1-DNA fraction was correlated with the GR-GRE interaction per se rather than with agonist activity in terms of transcription. This is further addressed below.

NF1-DNA binding correlates with GR-DNA binding but not with MMTV transcription. We previously showed that the GR-RU486 complex does not promote any MMTV transcription, chromatin remodeling, or nucleosome positioning but that it does promote a significant level of GR-GRE binding (6). The finding that RU486 supports both GR and NF1 binding indicates that NF1 binding occurs as a cooperative event driven by GR binding as such rather than by agonist-mediated chromatin remodeling (Fig. 3). To investigate this further, we

Scans are averages of each double lane, with indicated binding sites below. (C) Quantified DMS methylation of the two guanosines in the NF1 site, with double samples for the indicated lanes. (Right) The level of NF1 binding without hormone (bars A) is used as reference, i.e., $100 \%$ (double arrow). 
A.
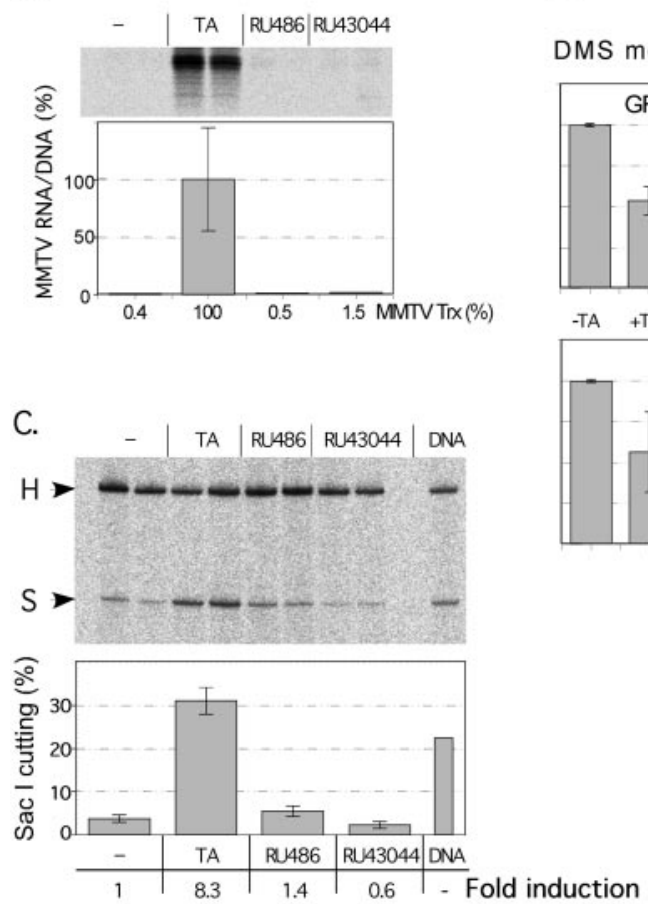

FIG. 4. NF1-DNA binding depends on ligand-induced GR-GRE binding also in the presence of the antagonist RU486. MMTV reporter DNA- and GR-NF1 mRNA-injected oocytes were treated with the indicated ligands for $10 \mathrm{~h}$. Two pools of 10 oocytes each were analyzed for transcription. (A) Diagram displaying the amount of mRNA expressed as a percentage of the agonist response. (B) DMS in vivo footprinting to quantify GR-GRE binding, an average of GRE II to IV (top), and NF1-DNA binding (bottom) from two pools of five oocytes each. (C) SacI in situ access analysis, showing the increase in cutting in relation to the mock-treated control. SacI bands (S) and HinfI bands $(\mathrm{H})$ are indicated on the left. Error bars signify the two values of the double samples.

studied the effects of NF1 DNA binding when the GR agonist or either of the two antagonists RU486 and RU43044 is added without prior agonist treatment. In agreement with previous results MMTV-driven transcription was strictly agonist dependent (Fig. 4A). Both antagonists generated only a background level of 0.5 to $1.5 \%$ of the transcription seen in the presence of the hormone agonist TA.

The DMS in vivo footprinting assay showed a distinct methylation protection, with 55\% methylation of the GR agonistbound GRE and $84 \%$ methylation by RU486-complex-bound GRE, while there was $94 \%$ methylation by the RU43044 antagonist-exposed GRE (Fig. 4B, top). This is in agreement with our previous results (Fig. 3) (6). The ligand-induced effect on NF1-specific guanine methylation protection was similar to that for GRE, i.e., 52, 84, and $93 \%$ for TA, RU486, and RU43044, respectively (Fig. 4B, bottom). This implies that $16 \%$ of the binding sites were occupied by their cognate factors in the presence of the antagonist RU486, both for the NF1 site and the GREs. This value represents about 29 and $31 \%$ of the binding seen in the presence of the agonist for GR and NF1 binding, respectively. This is in distinct contrast to the MMTV transcriptional response, where only a background level of transcription was seen with RU486.
Chromatin remodeling, as evaluated by an in situ SacI accessibility assay, showed an 8.3-fold increase in SacI cutting in the agonist-activated MMTV promoter compared to that for the untreated control oocytes (Fig. 4C). The antagonist-treated oocytes showed 1.4- and 0.6-fold effects on SacI cutting for RU486 and RU43044, respectively. The slightly stronger SacI cutting in the presence of RU486 than in the presence of RU43044 was seen in three independent experiments and is in concordance with the increased NF1 and GR binding shown by DMS in vivo footprinting (Fig. 4B) and the increase in SacI cutting by NF1 binding (Fig. 2C and D).

In agreement with the reversibility experiment (Fig. 3) the ligand-dependent fraction of NF1-DNA binding at the MMTV promoter is coupled to the GR-DNA interaction also when mediated by the antagonist RU486. We conclude that hormone-dependent NF1 binding is coupled to GR binding as such rather than to agonist-driven chromatin remodeling and transcription.

DNase I footprinting implies that NF1 enhances GR binding. DNase I in situ footprinting was utilized to address the histone-DNA organization over the MMTV GRE as well as transactive factor binding. By careful comparison of the DNase I cleavage pattern of naked DNA with that of the chromatinized template, a weak 10- or 11-bp ladder is seen both in the presence and absence of NF1 (Fig. 5A, compare lane 1 to lanes 2 and 6 and scans). This implies a preferred rotational positioning of the DNA along the histone octamer surface. The same rotational positioning was obtained before by in vitro nucleosome reconstitution of the same DNA sequence (36, 38 ). The addition of hormone resulted in weak DNase I protection over that for GREs I to IV (lanes 4 and 5 and scans). In the NF1 mRNA-injected oocytes there was also a distinct DNase I footprint over the NF1 binding site. This NF1-induced footprint was detectable also in the absence of hormone but was more distinct in its presence (compare lanes 6 and 7 to lanes 8 and 9 and scans). There was a hormone-dependent DNase I-hypersensitive site around position -66 in the oocyte extract lacking NF1 mRNA (lanes 4 and 5 and scans). The NF1induced footprint resulted in protection of this -66 hypersensitive site, and instead some further enhancement of DNase I cutting was seen around -55 (lanes 8 and 9 and scans).

Both DMS and DNase I footprinting showed that constitutive NF1 binding was further enhanced by the hormone-dependent GR binding. However, the DNase I footprinting also suggested that the protection of the GREs was more distinct in the NF1-containing oocytes (Fig. 5A, compare lanes 4 and 5 to lanes 8 and 9), except for a hypersensitive region at GRE IV (lanes 8 and 9) that might be caused by the NF1 binding. However, GR binds to GRE IV in spite of the hypersensitive DNase I pattern, as seen by DMS footprinting (Fig. 1C and 3B; see Fig. 6C). We also note that the overall DNase I cleavage pattern in the presence of hormone was more distinct in the presence of NF1 (see the DNase I pattern between the GRE I and GRE II in Fig. 5A, lanes 8 and 9).

MNase digestion revealed an NF1-dependent increase in the length of the hormone-activated subnucleosome B DNA and allowed its mapping. The B nucleosome of the MMTV promoter forms a subnucleosome complex of about 120 bp upon hormone activation, as revealed by probing with $\mathrm{B}$ nucleosome DNA after MNase digestion (5). To address the effects of NF1 
A.
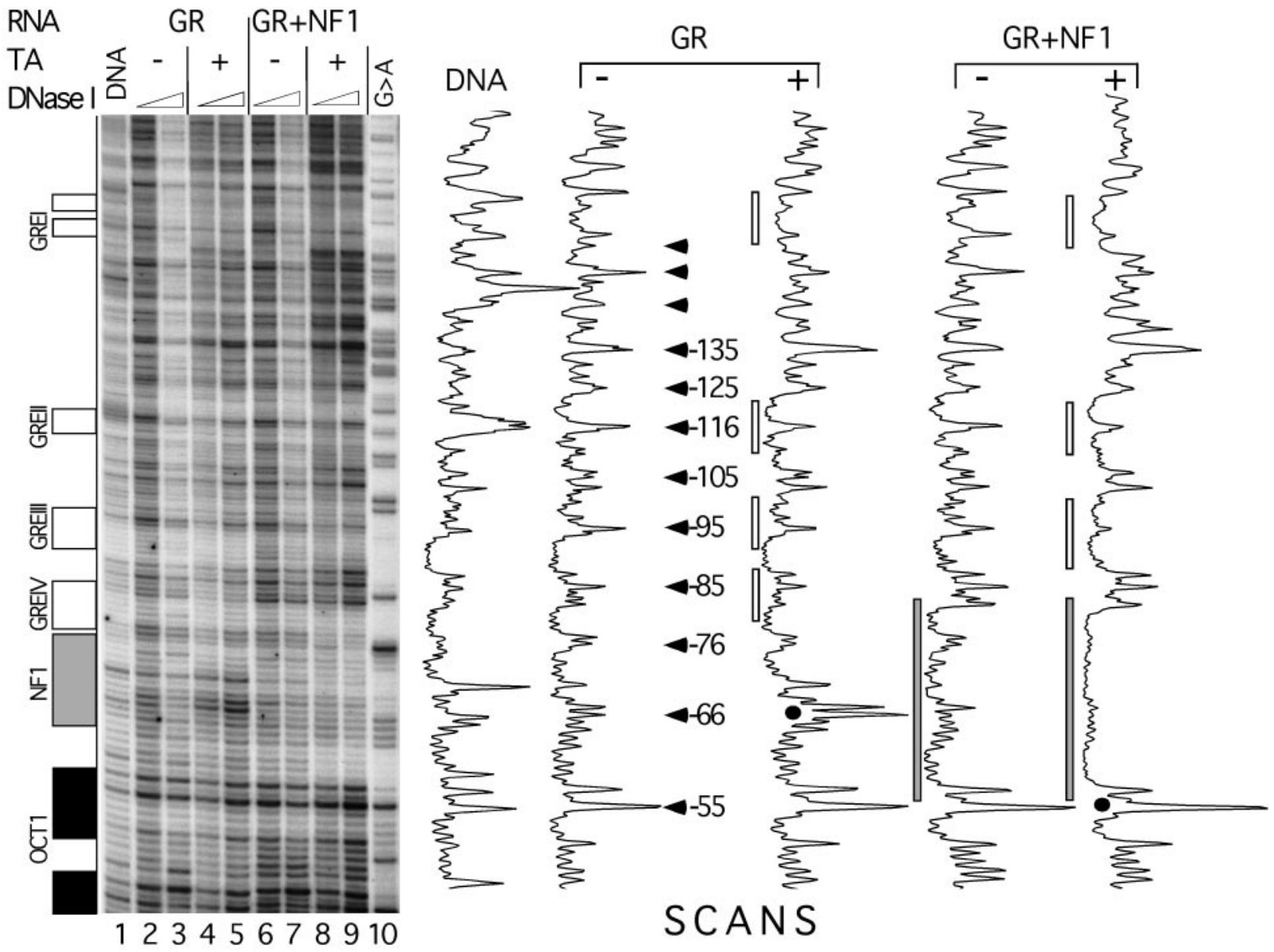

2345678910
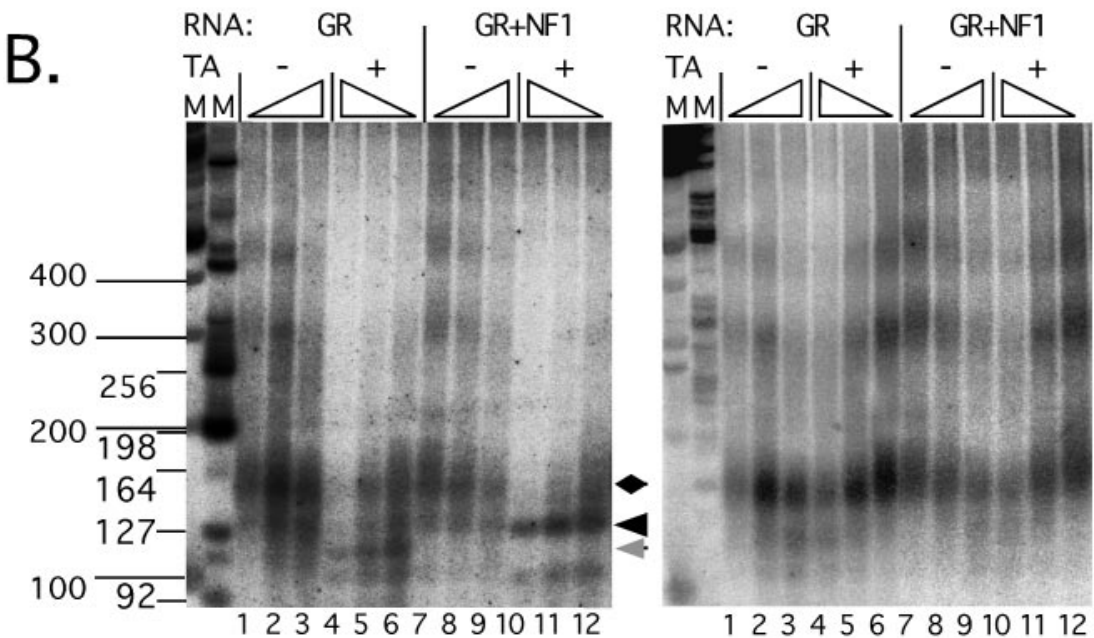

\section{B-nucleosome probe}

M13 vector probe

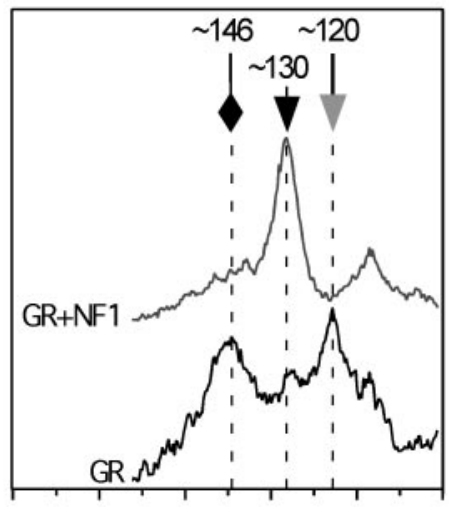

SCANS

FIG. 5. MMTV chromatin structure analysis using DNase I and MNase. Oocytes injected with the MMTV DNA reporter followed by GR mRNA with or without NF1 mRNA were either not treated $(-)$ or treated $(+)$ with hormone (TA). Pools of 10 oocytes were homogenized and divided in three aliquots for digestion with the indicated enzyme in increasing concentrations. (A) Primer extension of DNase I-digested samples with scans of one of the two displayed lanes for each oocyte sample. Lane DNA, naked DNA control; lane G $>$ A, DMS sequence ladder. Black 
on the B nucleosome structure, we analyzed the MNase digestion pattern on a $3.6 \%$ agarose gel, followed by electroblotting and probing with the DNA fragment from -218 to -54 , which covers the B nucleosome segment. The hormone-induced subnucleosome DNA of about $120 \mathrm{bp}$ is clearly seen (Fig. 5B, left, lanes 4 to 6 , and scan). The NF1-injected oocytes showed a 10to 15 -bp shift to a larger fragment (lanes 10 to 12 and scan). This increased length fits well with the NF1-induced protection of the hormone-induced - 66 DNase I-hypersensitive site and the concomitant increase of hormone-dependent cutting at -55 (Fig. 5A, compare lanes 5 and 9 and scans). These results, taken together, imply that the $3^{\prime}$ end of the subnucleosome B DNA segment in the presence of NF1 is located around -55 and that the $5^{\prime}$ end is located around the -185 position. Furthermore, they fit with the result of a previous effort to clone the MNase-induced subnucleosome DNA that generated a 119-bp MMTV DNA fragment containing the DNA sequence from -187 to -69 , which was isolated in the absence of NF1 (S. Belikov and Ö. Wrange, unpublished data). We conclude from these results that the $5^{\prime}$ end of the subnucleosomal DNA segment is located immediately distal to the strong GRE I at -185 to -171 (Fig. 1A). The MNase digestion also revealed that NF1 increased the DNA accessibility in the promoter region (Fig. 5B, compare lanes 4 to 6 with lanes 10 to 12). This is apparent from the faster MNase degradation kinetics caused by NF1. Importantly, NF1 generated an 130-bp subnucleosome that was significantly more MNase resistant than the 120-bp subnucleosome without NF1 (compare lanes 4 to 6 with lanes 10 to 12 and scans). The vector probe showed that the MNase-digested samples contain about the same amount of DNA in each lane, that the DNA is assembled into chromatin, thus generating a nucleosome ladder, and that the subnucleosomal fragments are specific for the MMTV promoter B nucleosome (Fig. 5B, right).

NF1 binds with an apparent 50-fold-higher affinity to the hormone-activated MMTV promoter and enhances GR binding. The possible interplay between GR and NF1 binding was further addressed by titrating the intracellular NF1 concentration during glucocorticoid-induced MMTV transcription (Fig. $6 \mathrm{~A}$ and $\mathrm{B}$ ). The amount of intranuclear GR protein was estimated to be $0.31 \mathrm{pmol}$ and the amount of NF1 protein was 0.26 pmol at the highest NF1 concentration. The amount of mRNA injected into oocytes was directly correlated with the amount of translated protein (C. Åstrand and O. Wrange, unpublished data). The volume of an oocyte nucleus was estimated as $40 \mathrm{nl}$ (24). Based on this the nuclear concentrations of GR and NF1 were calculated and correlated to DNA binding by DMS in in vivo footprinting (Fig. 6C and D).

There was a concentration-dependent protection of the NF1 site-specific guanines both in the presence and in the absence of hormone (Fig. 6C and D). Interestingly, a drastic increase in the hormone-dependent NF1 protection was revealed as the intranuclear NF1 concentration was increased from 80 to 240 $\mathrm{nM}$, while a more gradual concentration dependence of NF1 protection was obtained in the absence of hormone. Importantly, there was also a significant increase in the hormonedependent GRE protection induced by the DNA binding of NF1, i.e., at $>240 \mathrm{nM} \mathrm{NF1}$ as opposed to 0 to $80 \mathrm{nM} \mathrm{NF1,}$ where no significant DNA binding occurred. On the average ( \pm standard deviation) there was $51 \% \pm 7 \%$ methylation $(n=$ 4) of the GREs in the absence of NF1 binding and 37\% $\pm 6 \%$ methylation $(n=8)$ of the GREs in the presence of NF1 binding, $(P<0.005$; Student $t$ test) (Fig. 6D, bottom). The quantification of the DMS protection at all four GREs in the MMTV promoter revealed a parallel enhancement of GR binding by NF1 for all four GRE sites (data not shown). This positive effect of NF1 on GR binding corroborates the DNase I footprinting results (Fig. 5A).

The drastic increase in NF1-DNA binding allowed the apparent half-maximal binding to be estimated as occurring at about $160 \mathrm{nM}$ intranuclear NF1 protein, i.e., $\sim 6.2 \mathrm{fmol}$ of NF1 protein per oocyte nucleus. The same result was obtained in two independent experiments. There was a $\sim 50$-fold-higher concentration of NF1 required to reach the same level of DNA binding in the active promoter as compared to the hormoneinactived promoter (see also Discussion).

NF1 stimulates MMTV transcription when coexpressed with Oct1. The ubiquitous transcription factor Oct1 was previously shown to stimulate hormone-dependent $(9,41,47)$ and basal MMTV transcription in vivo $(10,47)$ and in vitro $(25)$. We addressed the effect of Oct1 in our system by injecting mRNA coding for the human Oct1 protein alone or in combination with NF1 and/or GR mRNA into Xenopus oocytes previously injected with the pMMTV:M13 reporter DNA. A representative experiment is presented (Fig. 7). As shown above, NF1 had no detectable effect on MMTV transcription, either in the presence or in the absence of hormone-activated GR. Oct1 alone had a minor effect on basal MMTV transcription, but Oct1 together with hormone-activated GR stimulated transcription 2.6-fold. Interestingly, NF1 in combination with Oct1 stimulated hormone-activated transcription to an even higher level, here 3.3-fold. Furthermore, NF1 and Oct1 coexpression in oocytes resulted in a significant constitutive MMTV transcription, hence confirming previous results by others $(10$, 47).

Similar results were obtained in three independent experiments. On the average transcription was stimulated 2.1-fold by Oct1 and 2.7-fold by Oct1 and NF1 in the presence of hormone-activated GR when hormone- and GR-dependent transcription was set to 1 . A paired Student $t$ test of these three experiments gave a significant stimulatory effect $(P<0.025)$ of NF1 together with hormone-activated GR and Oct1. There was no significant effect of NF1 alone on hormone- and GR-

arrowheads, 10- or 11-bp pattern of chromatin-specific hypercutting; black dots, hypersensitive sites; arrowheads with numbers, positions of chromatin-specific hypersensitivity relative to transcription start (+1); boxes, protected areas. (B) MNase-digested samples analyzed on a $3.6 \%$ agarose gel followed by electroblotting to a filter and probing first with a B nucleosome probe $(-218$ to -54 ; left) and then with the M13 vector probe (right). Size markers (base pairs) are given to the left. Black diamond, mononucleosome band of $\sim 146 \mathrm{bp}$; gray arrowhead, $\sim 120$-bp subnucleosome band (lanes 4 to 6); black arrowhead, 10-bp larger subnucleosome band induced by NF1 (lanes 10 to 12). Scans to the right are of lanes 5 (bottom) and 11 (top) of the B nucleosome-probed filter. 
A

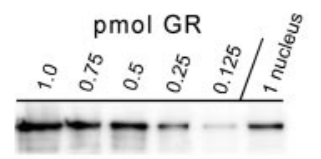

B.

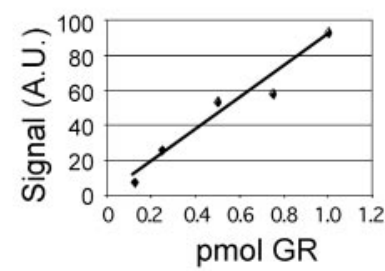

C.

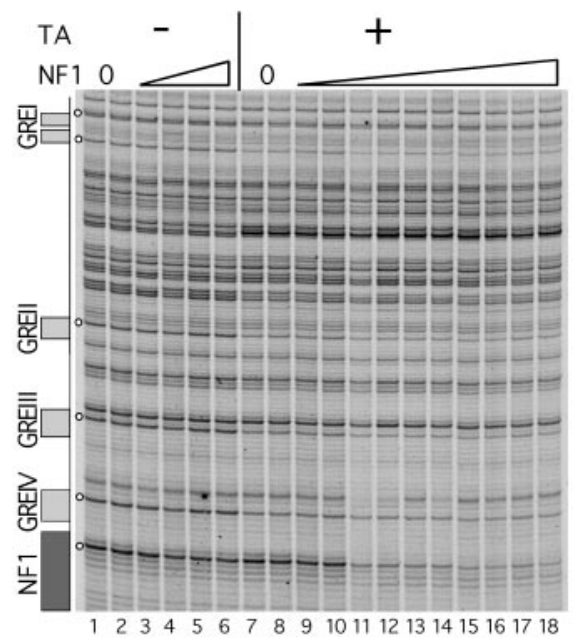

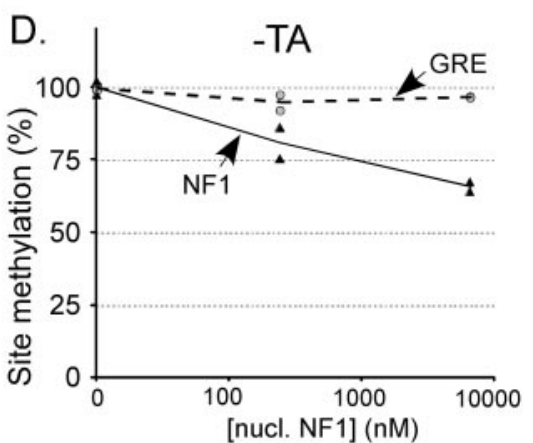

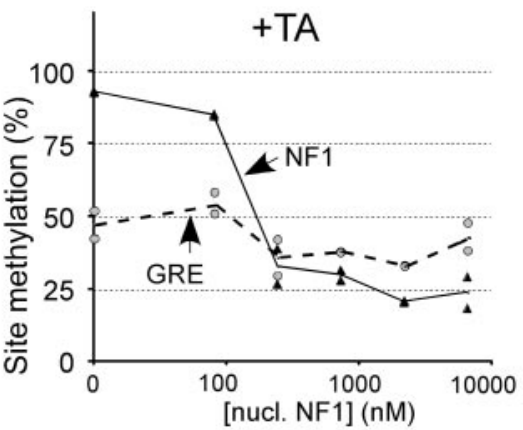

E.

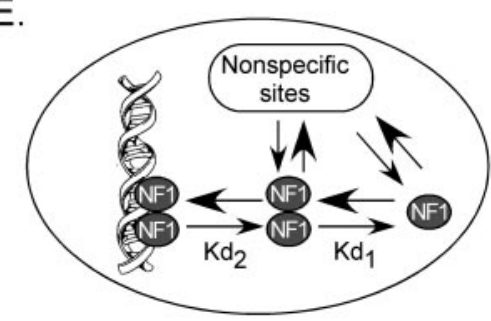

FIG. 6. Quantification of intranuclear GR and NF1 protein and specific DNA binding as a function of increasing concentrations of injected NF1 mRNA. Injection of MMTV DNA reporter was followed by injection of $5.8 \mathrm{ng}$ of GR mRNA also containing 0 to $5.8 \mathrm{ng}$ of NF1 mRNA prepared as a serial threefold dilution in five steps. Two pools of oocytes were analyzed for each NF1 mRNA concentration. (A) Western blot of purified rat GR and of nuclear GR from one nuclear equivalent of GR and NF1 mRNA-injected and hormone-treated oocytes. (B) Standard curve for GR quantification. (C) DMS in vivo footprinting of oocytes containing increasing intracellular NF1 protein not treated $(-)$ or treated $(+)$ with hormone (TA). Boxes and circles (left), protein binding sites and protected guanines, respectively. (D) Quantification of DMS methylation (from panel C) at the GREs, an average of GRE II to IV (circles, individual samples; dashed line, average), and at the NF1 binding site (triangles and solid line) as a function of NF1 protein concentrations. The intranuclear GR concentration was $7.8 \mu \mathrm{M}$ in the presence of hormone; the intranuclear NF1 concentrations were $0 \mathrm{nM}, 240 \mathrm{nM}$, and $6.5 \mu \mathrm{M}$ without hormone (lanes 1 to 6) and $0 \mathrm{nM}, 80 \mathrm{nM}, 240 \mathrm{nM}, 720 \mathrm{nM}, 2.2 \mu \mathrm{M}$, and $6.5 \mu \mathrm{M}$ with hormone (lanes 7 to 18). (E) Hypothetical model of NF1 homodimer formation and DNA binding.

dependent transcription, i.e., in the absence of Oct1 (Fig. 7 and data not shown).

We conclude that NF1 is transformed from a seemingly neutral factor into a stimulatory transcription factor when acting in the context of Oct1 and that this stimulatory effect is seen both in the presence and in the absence of hormone.

\section{DISCUSSION}

We demonstrate that there is an apparent 50-fold increase in NF1 binding affinity during hormone-induced MMTV transcription and that a significant and concentration-dependent fraction of the NF1 DNA sites are occupied by NF1 in the inactive MMTV promoter. Furthermore, the hormone-dependent fraction of NF1 binding is reversible, as shown by the addition of a hormone agonist followed by an antagonist. Un- expectedly, the hormone-dependent fraction of NF1-DNA binding is correlated with GR-GRE binding also in the presence of RU486 and is not directly correlated with transcription or agonist-driven chromatin remodeling. In addition GR and NF1 display cooperative DNA binding that generates a more MNase-resistant protein-DNA complex than that generated with GR alone. The GR- and NF1-containing enhanceosome complex organizes the -185 to -55 MMTV DNA. Finally, NF1 alone does not stimulate MMTV transcription, but when coexpressed with Oct1, NF1 does have a stimulatory effect on basal as well as hormone-dependent MMTV transcription.

NF1 binding to its DNA site in vivo. NF1 was previously shown to form a homodimer in the absence of DNA and to bind its cognate DNA site in this homodimeric form (26). Our demonstration of a narrow concentration range of the NF1 binding curve in the presence of hormone implies that an 

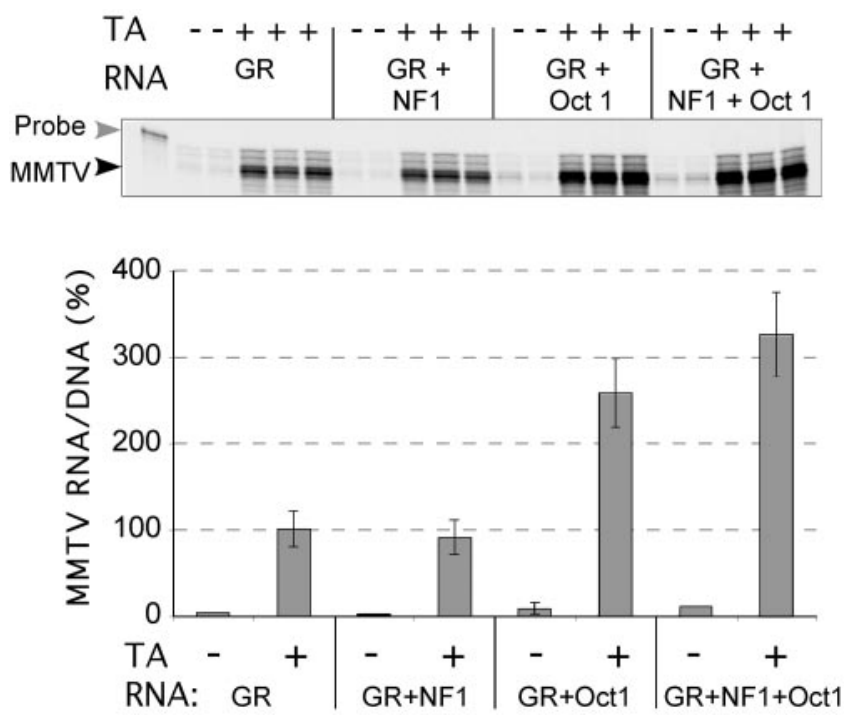

FIG. 7. NF1 stimulates MMTV transcription in the presence of Oct1. Oocytes were injected with 2 ng of pMMTV:M13 mixed with 0.4 ng of AdML reporter construct $18 \mathrm{~h}$ before hormone treatment and then with $3.8 \mathrm{ng}$ of GR and/or Oct1 mRNA and/or $0.4 \mathrm{ng}$ of NF1 mRNA as indicated $14 \mathrm{~h}$ before hormone treatment. Double samples without hormone and triple samples with hormone, 10 oocytes in each pool, were taken for RNA and DNA analysis. Hormone-treated oocytes containing GR only were used as reference $(100 \%)$. Error bars signify the $95 \%$ confidence interval. Student's $t$ test of the hormonetreated oocytes containing GR and Oct1 in the absence $(258 \% \pm 5 \%$ [mean \pm standard deviation]; $n=3)$ and presence $(325 \% \pm 3 \% ; n=$ 3 ) of NF1 rendered a $P$ value of 0.105 . The AdML RNA was not used as reference due to hormone- and protein context-dependent systematic variation.

important event occurs around 160 nM NF1, where half-maximal binding is seen (Fig. 6D). We hypothesize that this may reflect formation of the NF1 homodimer. The dissociation constant for NF1 binding to a DNA site with the same binding sequence as that of the MMTV NF1 site has been estimated to be about $10 \mathrm{nM}$ in an electrophoretic mobility shift assay-based DNA competition assay (44). We injected about 0.9 fmol of reporter DNA per oocyte. If this reporter DNA contains no more than the single NF1 site in the MMTV promoter per $10.25 \mathrm{~kb}$ of injected DNA, then this represents a capacity for specific binding of $0.9 \mathrm{fmol}$ of the NF1 protein at half-maximal saturation when NF1 is bound as a homodimer to DNA. This already accounts for about $15 \%$ of the total of $6.2 \mathrm{fmol}$ of intranuclear NF1 protein. It leaves the remaining $5.3 \mathrm{fmol}$ for the free-protein pool and for specific and nonspecific binding to other DNA sites. If all of the $5.3 \mathrm{fmol}$ of the NF1 protein were in the free pool, there would be a nuclear NF1 concentration of about $133 \mathrm{nM}$. However, the affinity of the homodimeric form of NF1 for DNA is expected to be much higher than that of the monomeric form. Hence it is conceivable that the vast majority of free NF1 is in monomer form at half-maximal DNA binding and that formation of the homodimer drives the DNA binding event. It is thus likely that the NF1 protein forms an equilibrium involving the monomeric and the dimeric forms at half-maximal DNA binding. The fractions of monomeric and dimeric NF1 occupied by nonspecific binding or by binding to cryptic DNA sites are not known, and hence we are unable to determine the dimerization and DNA-binding constants (Fig. 6E).

The mechanism of hormone-activated increase in NF1-DNA binding in a chromatin context. It is conceivable that an important function of chromatin is to restrict DNA accessibility in order to prevent basal transcription factors from binding to their cognate DNA sites and hence to avoid promoter leakage (23). An illustrative example of this chromatin effect is the enhanced occupancy of NF1 protein at the MMTV promoter during hormone activation (15). GR and NF1 binding experiments based on in vitro-reconstituted nucleosomes have demonstrated a compromised capacity for NF1-DNA binding in a nucleosomal context $(4,8,38)$. However, to the best of our knowledge this work represents the first attempt to quantify the effect of hormone activation on NF1 DNA site accessibility in vivo.

The intranuclear NF1 concentration at half-maximal NF1 binding in the presence and absence of hormone reflects the relative difference in DNA site accessibility in the active and the inactive promoters, respectively. We corrected for a background level of DMS methylation of about $21 \%$ based on the average methylation seen at the apparent NF1 site saturation of 2.17 to $6.5 \mu \mathrm{M}$ NF1 (Fig. $6 \mathrm{D}$, bottom). At $6.5 \mu \mathrm{M}$ intranuclear NF1 we reached $65 \%$ methylation, i.e., $35 \%$ occupancy in the absence of hormone (Fig. 6D, top). By extrapolating to the same level of occupancy in the hormone-activated state we obtain a $126 \mathrm{nM}$ NF1 concentration. The ratio of $6,500 \mathrm{nM} / 126$ $\mathrm{nM}$ indicates an apparent 50-fold increase in DNA site accessibility for NF1 by hormone activation of the MMTV promoter. This is only a crude estimation since we did not reach $50 \%$ binding in the absence of hormone and since we lack experimental data at $50 \%$ binding in the presence of hormone. However, we may conclude that a few percent of the NF1 sites will be occupied at the MMTV promoter also in the inactive state, provided that the intracellular NF1 concentration is in the range of $160 \mathrm{nM}$, i.e., the level required to reach halfmaximal saturation in the hormone-activated state. Hence it is not an all-or-none reaction when hormone activation increases NF1 accessibility but rather an increase from a significant basal level of NF1 binding. This may be of functional importance if another factor(s) is able to cooperate with NF1 in order to bind to neighboring DNA sites (see below).

The activation-dependent fraction of NF1 occupancy is dissociated when GR is removed by the addition of the strong antagonist RU43044. Our previous experiments show that the addition of an antagonist results in loss of the active chromatin structure, also including nucleosome positioning (6). This rearrangement of chromatin structure may well explain the loss of NF1 binding. Hence one may hypothesize that loss of GR binding results in chromatin folding back into an inactive conformation that decreases NF1 site accessibility to the level of that for the inactive promoter. The highly dynamic GR-GRE interaction, suggested by GR-green fluorescence protein fusion experiments (31), is expected to rapidly readjust its binding equilibrium when the agonistic ligand has been exchanged for an antagonist. The bound NF1, with its relatively high DNA binding affinity, does not seem to impose any major obstacle since it is also dissociated. It is conceivable that this is due to a loss of agonist-dependent chromatin remodeling at the MMTV promoter. However, the issue becomes more compli- 
cated by the only partial loss of NF1 binding in the presence of RU486. This antagonist was previously shown to partially support GR binding but without any detectable stimulation of transcription and without any detectable chromatin-remodeling effects (6) (Fig. 4). The close correlation between NF1 occupancy and RU486-dependent GR binding implies that a more direct cooperative effect is in operation, i.e., one without any requirement for agonist-mediated chromatin remodeling, at least in the case of the hormone antagonist RU486. The nucleosome structure was previously shown to mediate cooperative accessibility of various DNA binding factors $(1,3)$. An explanation for this might be that specifically bound proteins that have invaded a nucleosome may cause a propagation of altered histone-DNA contacts along the DNA axis and thus result in an increased DNA accessibility for any other protein to bind within the same nucleosome. The NF1 and GR binding cooperativity is corroborated by the finding of an increased GR binding in the presence of NF1 (Fig. 5A and 6D). Interestingly, the NF1-stimulated GR binding was seen for all four GR sites (I to IV) constituting the MMTV GRE (data not shown). Hence this NF1-mediated positive cooperativity is detectable within the entire B nucleosome. We propose that the mechanism of inherent cooperativity for transactive factor binding to nucleosomal DNA sites is relevant both for the GR-NF1 cooperativity and for the binding of GR to the cluster of GREs. The latter is in agreement with in vitro (36) and in vivo (48) experiments testing the binding of GR to the MMTV B nucleosome and may explain how GR can bind all sites in spite of an unfavorable rotational positioning of GRE II and GRE III on the histone octamer (36). A step-by-step invasion into nucleosome-organized DNA may be a common mechanism of enhanceosome assembly in vivo.

NF1 stabilizes the MMTV enhanceosome. The activated MMTV promoter digested with MNase was shown to contain a subnucleosomal structure of about $120 \mathrm{bp}$ (5) that essentially contains the B nucleosome sequence. Here we mapped this DNA segment based on the NF1-dependent increase in size to a 130-bp DNA fragment apparently involving the segment from -185 to -55 . This DNA contains the cluster of GREs previously shown to bind at least four GR homodimers and one homodimer of NF1. Hence $\sim 800 \mathrm{kDa}$ of DNA binding proteins is clustered within this DNA segment. It was previously suggested that the histone octamer (48) or a $\mathrm{H} 3 / \mathrm{H} 4$ tetramer (45) is still present within the GR-induced complex. If this is the case, then the molecular mass of this complex is $\sim 1$ MDa or more. We note that the DNA within the NF1-containing complex is more resistant to MNase digestion (Fig. 5B). This goes hand in hand with the binding cooperativity of NF1 and GR (Fig. 5 and 6) and implies that this complex forms a tight structural entity. Hence it is justified to refer to this complex as the hormone-induced enhanceosome. This structure might serve as a platform for recruitment of other factors involved in downstream events of transcription initiation.

The NF1 stimulates MMTV transcription only in the presence of Oct1. Previous studies have shown that NF1 stimulates hormone receptor-mediated MMTV transcription both in vitro (17) and in vivo $(9,11,12,40,47)$. Prado et al. (40) used Saccharomyces cerevisiae cells to demonstrate that hormoneinduced MMTV transcription is stimulated by the same porcine NF1-C1 protein as that used in our work and that a mutant MMTV promoter having a relaxed chromatin structure displayed a different mode of NF1 and GR synergy. It was thus astonishing to us that NF1 lacked significant effects on MMTV transcription in our experiments using Xenopus oocytes. The different results may be explained by differences in yeast and vertebrate chromatin structure or by differences in cofactor content in different cells. We, however, propose an alternative explanation. We established that NF1-dependent stimulation of transcription is seen only in the presence of Oct1. Hence the ubiquitously present Oct1 or another Oct1-like factor(s) might have been present in those cell types where NF1 was shown to stimulate hormone-activated MMTV transcription.

Oct1 was previously shown to enhance basal MMTV transcription in vitro $(25)$ as well as in vivo $(10,47)$ and also to stimulate hormone-induced transcription (9, 41, 47). Furthermore, in vitro binding experiments suggested that NF1 stabilizes the binding of Oct1 in the MMTV promoter (10). Mutational analysis implies that Oct1 directly contacts the GR DNA-binding domain and that this interaction serves as the basis for its strictly hormone-dependent recruitment of Oct1 to the MMTV promoter (41). Our finding of a cooperative transcriptional effect between NF1 and Oct1 both for basal and hormone-dependent transcription is in agreement with previous results.

Considering the tissue-specific distribution of the various NF1 gene products and their splice variants (21), one may speculate that the role of NF1 is to target relevant regulatory DNA segments and maintain tissue-specific expression, perhaps in order to counteract chromatin silencing activities. We note that NF1 and Oct1 caused a low but detectable MMTV transcription in the absence of hormone (Fig. 7). Hence NF1 seems to require the cooperation of other tissue-specifically expressed transactive factors such as Oct1, a member of the POU family transcription factors, which are also known to cooperate with many different transactive factors in gene regulation (37). We demonstrated a finely tuned balance between the intranuclear NF1 concentration and the DNA site occupancy. This suggests that cooperative interactions with other DNA-binding proteins can drastically and rapidly shift this equilibrium, perhaps in order to maintain a tissue-specific gene pattern in the active chromatin domain, i.e., to counteract chromatin-mediated silencing by effectuating a basal level of transcription. Further studies on the potential interactions between NF1 and Oct1 are needed to reveal their cooperative effects on chromatin structure and gene expression. Such studies are ongoing in our laboratory.

\section{ACKNOWLEDGMENTS}

This work was supported by the Swedish Cancer Foundation (project 2222-B03-19XCC) and Knut and Alice Wallenberg's foundation. P.-H. Holmqvist was supported by the Cell Signaling program of the Swedish Foundation for Strategic Research.

We thank U. Björk for skillful technical assistance and R. Morgenstern for helpful discussions concerning NF1 binding kinetics. We are grateful to L. Poellinger, M. Beato, and R. M. Gronostajski for providing cDNA coding for the human Oct1, the porcine NF1-C1, and the murine NF1-B2, respectively. We are grateful to K. Sousa for construction of the pigNF1-C1/RN3P plasmid. We thank B. Gelius at KaroBio for the generous gift of RU43044. 


\section{REFERENCES}

1. Adams, C. C., and J. L. Workman. 1995. Binding of disparate transcriptiona activators to nucleosomal DNA is inherently cooperative. Mol. Cell. Biol. 15:1405-1421.

2. Almouzni, G., and A. P. Wolffe. 1993. Replication-coupled chromatin assembly is required for repression of basal transcription in vivo. Genes Dev. 7:2033-2047.

3. Anderson, J. D., A. Thastrom, and J. Widom. 2002. Spontaneous access of proteins to buried nucleosomal DNA target sites occurs via a mechanism that is distinct from nucleosome translocation. Mol. Cell. Biol. 22:7147-7157.

4. Archer, T. K., M. G. Cordingley, R. G. Wolford, and G. L. Hager. 1991. Transcription factor access is mediated by accurately positioned nucleosomes on the mouse mammary tumor virus promoter. Mol. Cell. Biol. 11: 688-698.

5. Belikov, S., B. Gelius, G. Almouzni, and Ö. Wrange. 2000. Hormone activation induces nucleosome positioning in vivo. EMBO J. 19:1023-1033.

6. Belikov, S., B. Gelius, and O. Wrange. 2001. Hormone-induced nucleosome positioning in the MMTV promoter is reversible. EMBO J. 20:2802-2811.

7. Blomquist, P., S. Belikov, and O. Wrange. 1999. Increased nuclear factor 1 binding to its nucleosomal site mediated by sequence-dependent DNA structure. Nucleic Acids Res. 27:517-525.

8. Blomquist, P., Q. Li, and Ö. Wrange. 1996. The affinity of nuclear factor 1 for its DNA site is drastically reduced by nucleosomal organisation irrespective of its rotational or translational position. J. Biol. Chem. 271:153-159.

9. Brüggemeier, U., M. Kalff, S. Franke, C. Scheidereit, and M. Beato. 1991 Ubiquitous transcription factor OTF-1 mediates induction of the MMTV promoter through synergistic interaction with hormone receptors. Cell 64: 565-572.

10. Buetti, E. 1994. Stably integrated mouse mammary tumor virus long terminal repeat DNA requires the octamer motifs for basal promoter activity. Mol Cell. Biol. 14:1191-1203.

11. Buetti, E., and B. Kuhnel. 1986. Distinct sequence elements involved in the glucocorticoid regulation of the mouse mammary tumor virus promoter identified by linker scanning mutagenesis. J. Mol. Biol. 190:379-389.

12. Buetti, E., B. Kühnel, and H. Diggelmann. 1989. Dual function of a nuclear factor I binding site in MMTV transcription regulation. Nucleic Acids Res. 17:3065-3078.

13. Cato, A. C., P. Skroch, J. Weinmann, P. Butkeraitis, and H. Ponta. 1988 DNA sequences outside the receptor-binding sites differently modulate the responsiveness of the mouse mammary tumour virus promoter to various steroid hormones. EMBO J. 7:1403-1410.

14. Chaudhry, A. Z., A. D. Vitullo, and R. M. Gronostajski. 1998. Nuclear factor I (NFI) isoforms differentially activate simple versus complex NFI-responsive promoters. J. Biol. Chem. 273:18538-18546.

15. Cordingley, M. G., A. T. Riegel, and G. L. Hager. 1987. Steroid-dependent interaction of transcription factors with the inducible promoter of mouse mammary tumor virus in vivo. Cell 48:261-270.

16. das Neves, L., C. S. Duchala, F. Tolentino-Silva, M. A. Haxhiu, C. Colmenares, W. B. Macklin, C. E. Campbell, K. G. Butz, R. M. Gronostajski, and F. Godinho. 1999. Disruption of the murine nuclear factor I-A gene (Nfia) results in perinatal lethality, hydrocephalus, and agenesis of the corpus callosum. Proc. Natl. Acad. Sci. USA 96:11946-11951.

17. Di Croce, L., R. Koop, P. Venditti, H. M. Westphal, K. P. Nightingale, D. F Corona, P. B. Becker, and M. Beato. 1999. Two-step synergism between the progesterone receptor and the DNA-binding domain of nuclear factor 1 on MMTV minichromosomes. Mol. Cell 4:45-54.

18. Eisfeld, K., R. Candau, M. Truss, and M. Beato. 1997. Binding of NF1 to the MMTV promoter in nucleosomes: influence of rotational phasing, translational positioning and histone H1. Nucleic Acids Res. 25:3733-3742.

19. Gelius, B., P. Wade, A. P. Wolffe, Ö. Wrange, and A.-K. Östlund Farrants. 1999. Characterization of a chromatin remodeling activity in Xenopus oocytes. Eur. J. Biochem. 262:426-434.

20. Gelius, B., and O. Wrange. 2001. Glucocorticoid hormone-induced receptor localization to the chromatin fibers formed on injected DNA in Xenopus oocytes. Exp. Cell Res. 265:319-328.

21. Gronostajski, R. M. 2000 . Roles of the NFI/CTF gene family in transcription and development. Gene 249:31-45.

22. Grunder, A., T. T. Ebel, M. Mallo, G. Schwarzkopf, T. Shimizu, A. E. Sippel, and H. Schrewe. 2002. Nuclear factor I-B (Nfib) deficient mice have severe lung hypoplasia. Mech. Dev. 112:69-77.

23. Han, M., U.-J. Kim, P. Kayne, and M. Grunstein. 1988. Depletion of histone $\mathrm{H} 4$ and nucleosomes activates the PHO5 gene in Saccharomyces cerevisiae. EMBO J. 7:2221-2228.

24. Hausen, P., and M. Riebesell. 1991. The early development of Xenopus laevis, an atlas of histology. Springer-Verlag KG, Berlin, Germany.

25. Kim, M. H., and D. O. Peterson. 1995. Oct-1 protein promotes functional transcription complex assembly on the mouse mammary tumor virus promoter. J. Biol. Chem. 270:27823-27828.
26. Kruse, U., and A. E. Sippel. 1994. Transcription factor nuclear factor I proteins form stable homo- and heterodimers. FEBS Lett. 348:46-50.

27. Kutoh, E., P. E. Strömstedt, and L. Poellinger. 1992. Functional interference between the ubiquitous and constitutive octamer transcription factor 1 (OTF-1) and the glucocorticoid receptor by direct protein-protein interaction involving the homeo subdomain of OTF-1. Mol. Cell. Biol. 12:49604969.

28. Li, Q., and Ö. Wrange. 1995. Accessibility of a glucocorticoid response element in a nucleosome depends on its rotational positioning. Mol. Cell. Biol. 15:4375-4384.

29. Luisi, B. F., W. X. Xu, Z. Otwinowski, L. P. Freedman, K. R. Yamamoto, and P. B. Sigler. 1991. Crystallographic analysis of the interaction of the glucocorticoid receptor with DNA. Nature 352:497-505.

30. Maxam, A. M., and W. Gilbert. 1977. A new method for sequencing DNA. Proc. Natl. Acad. Sci. USA 74:560-564.

31. McNally, J. G., W. G. Muller, D. Walker, R. Wolford, and G. L. Hager. 2000 The glucocorticoid receptor: rapid exchange with regulatory sites in living cells. Science 287:1262-1265.

32. Mermod, N., E. A. O'Neill, T. J. Kelly, and R. Tjian. 1989. The proline-rich transcriptional activator of CTF/NF-1 is distinct from the replication and DNA binding domain. Cell 58:741-753.

33. Nagata, K., R. A. Guggenheimer, T. Enomoto, J. H. Lichy, and J. Hurwitz. 1982. Adenovirus DNA replication in vitro: identification of a host factor that stimulates synthesis of the preterminal protein-dCMP complex. Proc. Natl. Acad. Sci. USA 79:6438-6442.

34. Nowock, J., U. Borgmeyer, A. W. Püschel, R. A. W. Rupp, and A. E. Sippel. 1985. The TGGCA protein binds to the MMTV-LTR, the adenovirus origin of replication, and the BK virus enhancer. Nucleic Acids Res. 13:2045-2061.

35. Payvar, F., Ö. Wrange, J. Carlstedt-Duke, S. Okret, J.-Å. Gustafsson, and K. R. Yamamoto. 1981. Purified glucocorticoid receptors bind selectively in vitro to a cloned DNA fragment whose transcription is regulated by glucocorticoids in vitro. Proc. Natl. Acad. Sci. USA 78:6628-6632.

36. Perlmann, T., and O. Wrange. 1988. Specific glucocorticoid receptor binding to DNA reconstituted in a nucleosome. EMBO J. 7:3073-3079.

37. Phillips, K., and B. Luisi. 2000. The virtuoso of versatility: POU proteins that flex to fit. J. Mol. Biol. 302:1023-1039.

38. Pina, B., U. Bruggemeier, and M. Beato. 1990. Nucleosome positioning modulates accessibility of regulatory proteins to the mouse mammary tumor virus promoter. Cell 60:719-731.

39. Prado, F., R. Koop, and M. Beato. 2002. Accurate chromatin organization of the mouse mammary tumor virus promoter determines the nature of the synergism between transcription factors. J. Biol. Chem. 277:4911-4917.

40. Prado, F., G. Vicent, C. Cardalda, and M. Beato. 2002. Differential role of the proline-rich domain of nuclear factor 1-C splice variants in DNA binding and transactivation. J. Biol. Chem. 277:16383-16390.

41. Prefontaine, G. G., M. E. Lemieux, W. Giffin, C. Schild-Poulter, L. Pope, E. LaCasse, P. Walker, and R. J. Hache. 1998. Recruitment of octamer transcription factors to DNA by glucocorticoid receptor. Mol. Cell. Biol. 18: 3416-3430.

42. Richard-Foy, H., and G. L. Hager. 1987. Sequence-specific positioning of nucleosomes over the steroid-inducible MMTV promoter. EMBO J. 6:23212328.

43. Ringold, G. M., K. R. Yamamoto, G. M. Tomkins, J. M. Bishop, and H. E. Varmus. 1975. Dexamethasone-mediated induction of mouse mammary tumor virus RNA: a system for studying glucocorticoid action. Cell 6:299-305.

44. Roulet, E., P. Bucher, R. Schneider, E. Wingender, Y. Dusserre, T. Werner, and N. Mermod. 2000. Experimental analysis and computer prediction of CTF/NFI transcription factor DNA binding sites. J. Mol. Biol. 297:833-848.

45. Spangenberg, C., K. Eisfeld, W. Stunkel, K. Luger, A. Flaus, T. J. Richmond, M. Truss, and M. Beato. 1998. The mouse mammary tumour virus promoter positioned on a tetramer of histones $\mathrm{H} 3$ and $\mathrm{H} 4$ binds nuclear factor 1 and OTF1. J. Mol. Biol. 278:725-739.

46. Steele-Perkins, G., K. G. Butz, G. E. Lyons, M. Zeichner-David, H. J. Kim, M. I. Cho, and R. M. Gronostajski. 2003. Essential role for NFI-C/CTF transcription-replication factor in tooth root development. Mol. Cell. Biol. 23:1075-1084.

47. Toohey, M. G., J. W. Lee, M. Huang, and D. O. Peterson. 1990. Functional elements of the steroid hormone-responsive promoter of mouse mammary tumor virus. J. Virol. 64:4477-4488.

48. Truss, M., J. Bartsch, A. Schulbert, R. J. G. Hache, and M. Beato. 1995. Hormone induces binding of receptors and transcription factors to a rearranged nucleosome on the MMTV promoter in vivo. EMBO J. 14:17371751 .

49. Zaret, K. S., and K. R. Yamamoto. 1984. Reversible and persistent changes in chromatin structure accompany activation of a glucocorticoid-dependent enhancer element. Cell 38:29-38.

50. Zernika-Goetz, M., J. Pines, K. Ryan, K. R. Siemering, J. Haseloff, M. J. Evans, and J. B. Gurdon. 1996. An indelible lineage marker for Xenopu using a mutated green fluorescence protein. Development 122:3719-3724. 